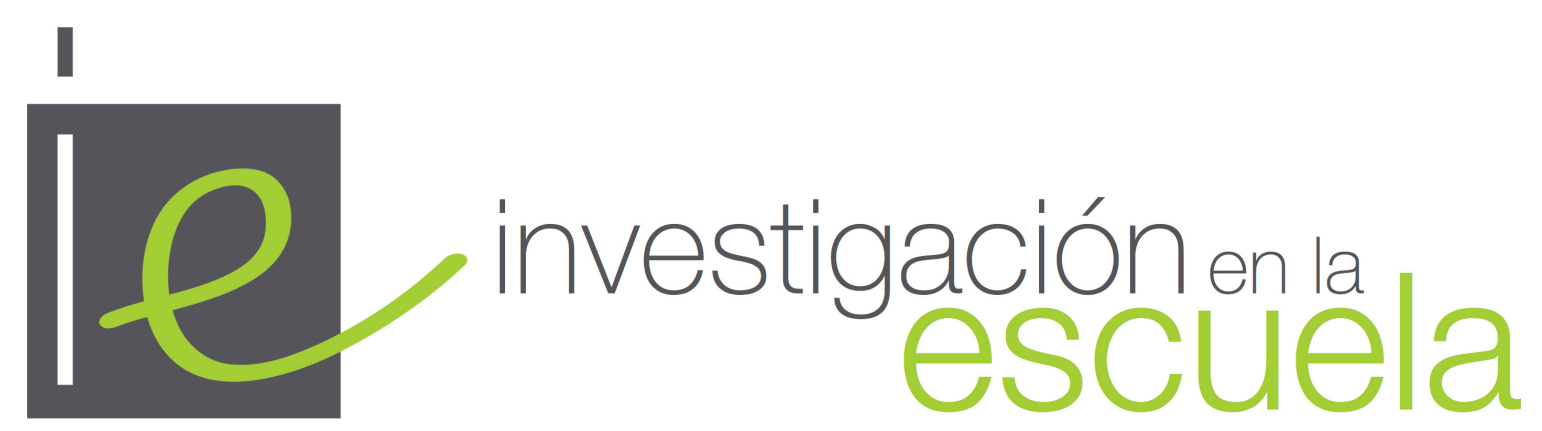

Revista internacional de investigación e innovación educativa

Número 100

30 de abril de 2020

ISSN 2443-9991

\title{
Motivos para comprometerse, desvincularse y revincularse con los estudios superiores
}

\author{
Daiana Rigo ${ }^{1}$, Melina Irusta ${ }^{2}$, Gisela Bechero ${ }^{2}$ y Stefania Amaya ${ }^{1}$ \\ Universidad Nacional de Río Cuarto ${ }^{1}$ y Centro Educativo Terapéutico Kalen ${ }^{2}$ \\ Argentina
}

Citación: Rigo, D., Irusta, M., Bechero, G. y Amaya, S. (2020). Motivos para comprometerse, desvincularse y revincularse con los estudios superiores. Investigación en la Escuela, 100, 71-87. DOI: https://dx.doi.org/10.12795/IE.2020.1100.06

Resumen: La realidad de las aulas universitarias, a día de hoy, continúa ofreciendo incógnitas acerca de su impacto en el desarrollo de los procesos de implicación de los estudiantes. Por eso, el estudio desarrollado tuvo como objetivo describir los motivos que los estudiantes universitarios construyen para comprometerse con sus estudios superiores, desvincularse o revincularse con los procesos de aprendizaje. Se estudia una muestra de 150 alumnos, seleccionados de manera no aleatoria, matriculados en la Universidad Nacional de Río Cuarto, Argentina. Se recolectan datos con un cuestionario y entrevistas semiestructuradas. Los resultados muestran que las construcciones son individuales pero compartidas. Resaltando la importancia de recrear los contextos educativos, expandir el aula, crear lazos con la comunidad, brindar apoyos a los estudiantes frente a las dificultades que atraviesan durante el cursado y apostar a la formulación de metas desde la agencia personal que permitan trazar el recorrido académico con compromiso. Lo encontrado posibilita generar pautas para futuras experiencias educativas para involucrar a los estudiantes con el proceso educativo.

Facebook: Investigación en la Escuela

Artículo recibido: 06-04-2020

Twitter: @invescuela

Revisiones recibidas: 10-04-2020

Aceptado: 15-04-2020

DOI: https://dx.doi.org/10.12795/IE.2020.1100.06 
Palabras clave: "Enseñanza superior"; "clima de la clase”; "participación de los estudiantes"; "interés"; "ambiente socio-cultural"; "comunidad"; "relaciones interpersonales"; "afectividad".

\section{Reasons to engage, disassociate and relink with higher education}

Abstract: The reality of university classrooms, to this day, continues to offer unknowns about their impact on the development of student engagement processes. The study developed aimed to describe the reasons that university students build to engage with their higher education, disassociate themselves or relink with learning processes. A sample of 150 students is studied, selected nonrandomly, enrolled at the National University of Río Cuarto, Argentina. Data is collected with a questionnaire and semi-structured interviews. The results show that the constructs are individual but shared. Emphasizing the importance of recreating educational contexts, expanding the classroom, building ties with the community, supporting students in the face of the difficulties they face during the course, and betting on the formulation of goals from the personal agency that to chart the academic journey with commitment. Found makes it possible to generate guidelines for future educational experiences to engage students with the educational process.

Key words: "Higher education"; "classroom climate"; "student participation"; "interest"; "sociocultural environment"; "community"; "interpersonal relations"; "affectivity".

\section{Raisons de s'engager, de se dissocier et de se reconnecter à l'enseignement supérieur}

Resumè: La réalité des salles de classe universitaires, à ce jour, continue d'offrir des inconnues quant à leur impact sur le développement des processus d'engagement des étudiants. L'étude développée visait à décrire les raisons pour lesquelles les étudiants universitaires s'engagent dans leurs études supérieures, se dissocient ou reconnectent avec les processus d'apprentissage. Un échantillon de 150 étudiants est étudié, sélectionnés de manière non aléatoire, inscrit à l'Université nationale de Rio Cuarto, Argentine. Les données sont recueillies à l'inse où se trouve un questionnaire et des entrevues semi-structurées. Les résultats montrent que les constructions sont individuelles mais partagées. Soulignant l'importance de recréer des contextes éducatifs, d'élargir la salle de classe, d'établir des liens avec la communauté, de soutenir les élèves face aux difficultés qu'ils rencontrent pendant le cours, et de parier sur la formulation d'objectifs de l'organisme personnel qui tracer le parcours académique avec engagement. Trouvé permet de générer des lignes directrices pour les expériences éducatives futures pour engager les étudiants dans le processus éducatif. Mots-clés: "L’enseignement supérieur"; "le climat en classe"; "la participation des élèves"; "l’intérêt"; "l'environnement socioculturel"; "la communauté"; "les relations interpersonnelles"; "l'affectivité".

\section{Introducción}

Hablar de compromiso académico, se ha vuelto materia obligatoria entre quienes están preocupados por la calidad educativa, las elevadas tasas de abandono y deserción. Los resultados de las investigaciones sobre este constructo son prometedores, en tanto evidencian el impacto que tiene sobre el rendimiento académico, la finalización de los estudios y los niveles de asistencia (CasusoHolgado et al., 2013; Montes Ruiz, 2016; Rodríguez et al., 2014). Motivados en contribuir a mejorar el compromiso de los estudiantes, en el presente estudio nos focalizamos en describir los motivos que los estudiantes universitarios construyen para comprometerse, así como las razones y los comportamientos que despliegan cuando se desvinculan y vuelven a reengancharse.

El par compromiso - desvinculación académica, tiene distintos tratamientos en la literatura científica. Por un lado, se lo define como opuestos, aludiendo a la necesidad de generar indicadores 
empíricos distintos para medir cada uno (Reeve, Cheon \& Jang, 2019); por otro lado, se lo entiende como un continuo, en tanto, son los mismos comportamientos los que se mueven bajo unos mismos criterios con mayor o menor presencia en los estudiantes más o menos comprometidos (Hofkens y Ruzek, 2019).

Posicionados en esta última tendencia, se comprende que el compromiso es un metaconstructo dinámico, flexible y modificable. A las clásicas definiciones de compromiso, como la inversión psicológica que hace el sujeto hacia los estudios (Newmann, Wehlage \& Lamborn, 1992); el grado de implicación con las tareas académicas; la intensidad o emoción con la cual los sujetos se conectan para iniciar y desarrollar tareas de aprendizaje o la participación que conecta a la persona con la actividad (Appleton, Cristenson, Kin \& Reschly, 2006), se suma recientemente, la importancia de reconocer que los estudiantes dirigen su participación por objetivos y propósitos específicos, entre los cuales, se mencionan, conseguir mejores logros educativos, satisfacer sus motivaciones y crear entornos de aprendizaje favorables y afines a sus intereses (Reeve, Cheon \& Jang, 2019).

Definición que, no sólo recupera la idea del compromiso como un metaconstructo integrado por tres dimensiones, afectiva, cognitiva y conductual, sino que además incorpora la agencia como la cuarta faceta proactiva del término. Rescata, asimismo, la interacción del sujeto con el entorno, mediada por metas educativas que moldean el compromiso de los estudiantes.

La dimensión cognitiva incluye el uso de estrategias personalizadas que utiliza el estudiante ante el aprendizaje y para regular la toma de decisiones (Reeve, 2012; Rigo y Donolo, 2019).

La conductual, refiere a la participación del alumno en las actividades educativas y sus conductas observables en el aula ligadas al esfuerzo y el logro (Connell, 1990; Finn, 1989; Fredricks, Blumenfeld \& Paris, 2004; Martin, 2008; Skinner, Kinderman \& Furrer, 2009). Mientras que, la afectiva, se asocia con el grado de identificación de los alumnos con la escuela, las actitudes hacia el ámbito escolar, el aprendizaje, sus relaciones con los profesores, los compañeros y otros profesionales de la institución educativa (Brigman et al., 2015; Voelkl, 1997). Un buen indicador de compromiso emocional es el interés hacia el trabajo en el aula; a la inversa, menor implicación afectiva sería afín a conductas tales como el aburrimiento, la ansiedad, la frustración y los nervios en el aula (Rigo y Donolo, 2019).

De acuerdo a últimas investigaciones, se ha añadido una nueva dimensión denominada agente, que se define como la habilidad del alumno para establecer metas propias y constructivas en el proceso activo del aprendizaje. En este sentido, la dimensión agéntica se refiere a la manera de comportarse, sentir y pensar de manera autónoma para modificar el contexto instructivo en pos de los objetivos y metas educativas personales (Wang \& Peck, 2013).

A través de esta última dimensión del compromiso, los estudiantes encuentran formas de enriquecer, modificar y personalizar su instrucción. Por lo tanto, este tipo de compromiso está vinculado a las contribuciones constructivas y transaccionales, aquellas que modifican, enriquecen y personalizan el aprendizaje de los estudiantes en el aula (Bandura, 2006). Los estudiantes comprometidos en esta dimensión demuestran iniciativa y colaboración, creando momentos de aprendizaje en el aula que contribuyen directamente a sí mismos (por ejemplo, apoyo motivacional y logros) y al propio ambiente de la clase (comunicación profesor-alumno). Bandura (2006) sostiene que la agencia humana implica la intencionalidad con los planes y las estrategias que podrían requerir el acomodamiento de intereses personales con los de otros agentes, por ejemplo, con los profesores. Por lo tanto, la interacción docente-alumno es un factor clave para generar experiencias significativas en el aula.

La ausencia o niveles bajos en cada dimensión ha llevado a varios autores a describir un nuevo fenómeno presente en las instituciones educativas que conceptualizan como desmovilización, desenganche o desvinculación de un sector creciente de estudiantes con respecto a los estudios. La desvinculación educativa debe entenderse como un proceso, una trayectoria, evitando análisis 
simples que lo conceptualicen como un hecho aislado, donde el abandono es el punto de clímax del proceso (Fernández Enguita, Mena y Riviere, 2010; Montes Ruiz, 2016). Se produce por la acumulación de elementos que originan cierto grado de alejamiento respecto a la escala de valores, pautas de actuación o los símbolos de identificación con la institución educativa. El desenganche, por lo tanto, implica problemas distintos y no necesariamente es intencional por parte del alumno, sino que está influenciado, a su vez, por su entorno más próximo. Existen actitudes y acciones en el aula, que muestran algunos indicios de este proceso, tales como el absentismo, la falta de respuesta académica, la apatía frente a los nuevos aprendizajes, la falta de disciplina (Martínez, 2007, 2009), o la percepción de considerar que todo lo vinculado con su educación es una experiencia tediosa e irrelevante para su formación y vida futura (Finn, 1993).

Lo sorprendente es que este tipo de problemática alcanza también a los niveles de educación superior, donde los estudiantes en principio han elegido estar voluntariamente. En este caso, Coulon (1999) refiere a una situación de 'no afiliación’ con la institución universitaria. Y otros han destacado que la complejidad creciente, tanto de la sociedad en general, como de la misma universidad, ha contribuido a generar un tipo de estudiantes desorientados y poco motivados por los estudios que, o bien abandonan relativamente temprano, o bien disminuyen su participación y asistencia a las actividades académicas (Martínez y Salanova, 2003). Al respecto, Montes Ruiz (2016), menciona que la desvinculación, tanto afectiva como conductual y cognitiva, genera trayectorias educativas signadas por la falta de interés o participación académica.

Astin (1993), Pascarella y Terenzini (2005) postulan que la decisión del estudiante de permanecer o abandonar sus estudios, está influida por su grado de interacción académica, social y a su vez, condicionada por las actividades educativas con propósitos definidos que se diseñan para incrementar la posibilidad de que los estudiantes aprendan y logren avances cognitivos significativos. La decisión de desvincularse o revincularse, está atravesada por factores relacionados al plano personal, el nivel de apoyo afectivo que tengan a nivel familiar o de sus referentes cercanos respecto a continuar los estudios, el entramado de redes sociales tejidas en torno al ámbito académico, su trayectoria escolar y educativa, las características propias del programa de estudio y la institución educativa donde lo cursan (Rodríguez et al., 2014). Montes Ruiz (2016), identifica, que tanto los procesos de toma de decisión, como los procesos derivados del contexto social impactan sobre los procesos de vinculación y desvinculación académica.

Por su parte, Graumann (1971) identifica como factores de desvinculación, las capacidades, las características de las tareas y los impulsos de activación, tales como, motivación, expectativas e intereses. En este sentido, los planes de estudios, las escasas opciones de trabajo al finalizar la carrera universitaria o terciaria, o la ausencia de motivaciones intrínsecas al aprendizaje producen estados de desmotivación en el alumnado y, consecuentemente, aumenta el número de estudiantes con poco compromiso. Son esos mismos factores situacionales y personales en positivo, lo que motivan a los estudiantes a revincularse con los estudios superiores (Montes Ruiz, 2016).

Claramente, son procesos, compromiso, desvinculación y revinculación, que necesitan nuevos estudios para dar respuestas ajustadas al contexto educativo en cuestión.

\section{Metodología}

La presente investigación, de diseño cualitativo, se propuso describir los motivos que, estudiantes universitarios, encuentran para comprometerse, desvincularse o revincularse con los estudios superiores. 


\section{Muestra}

Participaron 150 estudiantes universitarios, cuya selección fue por conveniencia, inscriptos en la carrera de Licenciatura en Psicopedagogía de la Facultad de Ciencias Humanas, Universidad Nacional de Río Cuarto, Argentina. En relación a las características de la muestra, el 98\% de los estudiantes participantes son mujeres. Asimismo, el 48\% se encontraban cursando segundo año del plan de estudio, un 20\% cursaban el tercer año, otro $20 \%$ estaba en cuarto año y el $12 \%$ restante estaba en quinto año. Todos los sujetos ofrecieron el consentimiento informado por escrito.

\section{Instrumento}

Se utilizó un protocolo con preguntas abiertas para indagar sobre el compromiso académico desde la perspectiva del estudiante universitario. El instrumento indagaba sobre 8 dimensiones vinculadas a factores contextuales que Ríos, Bozzo, Marchant y Fernández (2010), relacionan con la configuración del aula por parte del docente para favorecer el compromiso, como así también, para comprender las características de aquellas clases que favorecen o no la implicación de los alumnos. Esas dimensiones fueron: las tareas de aprendizaje, relaciones entre pares y con docentes, contenido enseñado, modalidades de evaluación, recursos tecnológicos, apoyos extraclase -consultas-; a partir de las cuales se le solicitaba al estudiante describir aquellos aspectos, comportamientos o acciones que los comprometían y no tanto con las clases universitarias. Asimismo, contaba con otras dos secciones adicionales, para recuperar información sobre conductas o decisiones frente a situaciones de vinculación y desvinculación ligadas a los estudios superiores. Cada dimensión contempló dos preguntas abiertas y las secciones solo una.

El instrumento fue completado por escrito por 120 estudiantes y el resto lo hizo en modalidad de entrevista semiestructurada para obtener respuestas más profundas e indagar más en detalle los motivos mencionados.

\section{Análisis de datos}

Se utilizó el método de comparación constante, el cual consiste en recoger, codificar y analizar los datos para generar y crear diferentes categorías (Vasilachis, 2007). En este proceso, se llevó adelante una triangulación entre investigadores desde diferentes perspectivas del mismo fenómeno. Del análisis integrado surgieron 2 categorías que responder el objetivo inicial del estudio.

\section{Resultados}

A continuación, se presentan los resultados encontrados en el presente estudio, los cuales se organizan en dos grandes categorías.

\section{Motivos para comprometerse}

En esta categoría se incluyen recursos y contextos que los estudiantes expresan como más afines a sus intereses. Tanto la extensión del clima del aula hacia nuevos escenarios educativos vinculados con el futuro quehacer profesional, como el uso de tecnologías de la comunicación y de la información (TIC) para hibridar los contextos tradicionales de enseñanza, son señalados como entornos que comprometen por sus dinámicas y manera de interacción entre pares, docentes y contenido.

Los estudiantes, sostienen que no sólo se aprende en espacios formales de educación, sino que, diversos ambientes ligados a su formación se vinculan con la construcción de conocimientos, sin ser el aula el único espacio. En ellos los sujetos despliegan sus comportamientos afectivos y 
conductuales, que dan cuenta de su compromiso con los estudios académicos en contextos extraáulicos, como otra alternativa al aula, en tanto los perciben como experiencias gratificantes.

"Una experiencia educativa que me pareció muy rica y sumamente motivadora fueron las prácticas que se llevaron a cabo (...) durante el cuarto año de la carrera (...). En cuanto al aprendizaje teórico me ayudó muchísimo ya que era una materia con gran carga teórica un tanto difícil de comprender y con la bajada de la teoría a la realidad logre sortear este obstáculo" (S11).

“...particularmente las prácticas socio comunitarias (...) resultaron ser una experiencia educativa muy rica e interesante, que permitió en primer lugar vivir una experiencia distinta, fuera de la universidad, compartiendo con personas de diversos ámbitos, y en segundo lugar facilito mi aprendizaje en cuanto a los contenidos brindados por cada una de las cátedras" (S12).

"Una experiencia que fue interesante para mí fue la realización de las prácticas sociocomunitarias (...). Disfruté mucho realizándolas y me gustaba participar en este proceso. Considero que me dejó una experiencia y un aprendizaje muy positivo, no sólo porque me permitió vincular lo teóricamente aprendido haciéndolo, sino que además la cátedra siempre hizo presente el objetivo de las prácticas; siempre nos recordó por qué estábamos ahí y qué era importante hacer..." (S26).

"Una experiencia educativa que me parece muy interesante destacar es la de las Prácticas Profesionales en Educación (...) creo que es muy importante e interesante realizar las prácticas, deja muy buenos aprendizajes, después de cinco años de ver todas las materias y de estudiar todo lo teórico salir a la práctica y ver lo que realmente está pasando en las escuelas es una muy buena experiencia..." (S6).

"Una de las experiencias educativas que más me brindó fue la práctica profesional realizada en el último año de la carrera, ya que aprendí muchísimo junto a los niños y profesionales con los que trabajé. Fue una experiencia muy rica en cuanto a conocimientos y en cuanto a lo personal, sobre todo, ya que me inserté en un ámbito donde no había trabajado nunca..." (S22).

Son contextos que responden a una estrategia educativa, al interior de un plan de estudio, pensadas y diseñadas para aportar y enriquecer el proceso de formación profesional de los estudiantes. Específicamente, las Prácticas Socio-Comunitarias (PSC) y Profesionales (PP) aparecen en las voces de los estudiantes, quiénes rescatan a tales entornos, no sólo por los materiales, el equipamiento o infraestructura que los conforman, sino por las interacciones entre el saber teórico y los desafíos prácticos.

Macchiarola, Martini y Montebelli (2012), referentes de las PSC, mencionan que el aprendizaje que allí se construye es situado, es un conocimiento que se produce en y desde la acción, en interacción con otros. Son experiencias en acción, que buscan andamiar el proceso de enseñanza y aprendizaje, tanto para los alumnos, como para los miembros de la comunidad, promoviendo la toma de dicciones y acciones de manera autónoma, a partir de la curricularización de experiencias pre profesionales en territorio, en vista a promover la formación integral con el compromiso con la sociedad (Cecchi, Pérez y Sanllorenti, 2013).

De Souza Santos (2005), utiliza el término “ecología de saberes”, para describir a aquellas prácticas que en pos del diálogo entre saberes y actores diversos, comparten y construyen un conocimiento común en una relación de horizontalidad. Ese saber lo encuentran los estudiantes también en las PP que, por lo general, tienen lugar sobre la finalización de la carrera, a partir de las cuales logran encontrar el valor de utilidad del conocimiento teórico adquirido a lo largo de la carrera, otorgándole otro sentido, contextualizado y situado. 
"Contextos como hacer observaciones en contextos reales donde en el futuro puede ser un posible ámbito de trabajo, las prácticas socio-comunitarias, ateneos con profesionales de psicopedagogía" (S1).

"Son otros espacios que ayudan a que podamos establecer esa vinculación teoríapráctica y también actuar como futuros psicopedagogos" (S8).

"Otros contextos en donde haya interacción entre los alumnos-profesores, donde lo teórico puede aplicarse a lo práctico, por ejemplo, las prácticas socio-comunitarias" (S11).

"Fue una experiencia rica en varios sentidos, nos acercamos a un posible ámbito de desempeño para las psicopedagogas que no conocíamos, por otro vivenciamos realidades muy diferentes a las que estábamos acostumbradas a compartir” (S13).

Colás Bravo, González Ramírez y Reyes de Cózar (2015), entienden que uno de los principales desafíos de la educación es ayudar a los estudiantes a comprender el mundo y la manera en que las cosas ocurren, a ser conscientes de ellas, promoviendo prácticas integradas a la comunidad o trabajos académicos que guarden relación con problemas de la vida real, y potencien su compromiso.

Paralelamente, el grupo de estudiantes de la presente investigación señalan que, los videos y las clases virtuales, ofrecen mayores posibilidades para relacionar la teoría con la práctica, así como dar sentido a los contenidos y promover un aprendizaje contextualizado y significativo. En voces de los estudiantes se expresa:

"... compartir videos y con ellos discutir luego; ;me enganchan las clases virtuales son interesantes!” (Estudiante 25, C.G.).

"Me parece que sí, hay estrategias que son más favorables para nuestro compromiso con el aprendizaje, puede ser el uso de las tecnologías que hoy en día se utilizan mucho, el uso de los videos, imágenes, son estrategias que nos ayudan a comprender mejor" (S29). "Creo que esas clases didácticas apuntan a la participación del estudiante porque a partir de los videos, los estudiantes pueden hacer preguntas o si no entienden algo, lo consultamos también (...) clases más didácticas nos favorecen en ese sentido, porque nos entusiasman con la materia o con el tema, y también nos ayudan para la participación porque no es lo mismo que un profesor este parado al frente y que hable todo él" (S32).

“(...) Por ejemplo hacer un grupo en Facebook y a partir de allí dar una clase virtual o dar alguna actividad que se realice en la plataforma me parece que ayuda porque es una manera diferente de dar clases, es una manera alternativa y es algo innovador que yo particularmente lo que es tecnología, innovador y diferente me encanta por un interés particular mío y soy curiosa entonces esas actividades me parecen mucho más lindas que otras que tal vez hay que hacer un trabajo..." (S23).

“(...) El aula virtual también me parece una buena estrategia de aprendizaje” (S13). “(...) Utilizan los trabajos en grupos muchos, por que como te decía antes ayudan y favorece en el aprendizaje porque se intercambian opiniones, también los foros dentro del aula virtual o las clases extras que son con el objetivo de explicarnos y ayudarnos en los temas más difíciles que no se hayan entendido" (E, Sujeto $\mathrm{N}^{\circ} 6$ ).

Estos recursos son reconocidos por los estudiantes como apoyos instrumentales para profundizar sobre la comprensión de los contenidos y lecturas propuestas por las asignaturas o generar nuevas búsquedas para ampliar los conceptos desde nuevas posturas o autores. Windham (2005) puntualiza que, cualquier actividad que procure generar compromiso en los estudiantes debe incluir espacios para la interacción online, la exploración en la web, la vinculación social, así como, la inclusión de recursos multimedia, sin descuidar la instrucción docente sobre cómo navegar o 
investigar en las bibliotecas modernas -bases de datos de revistas en línea-. Componentes amigables con la nueva generación de estudiantes, quiénes se caracterizan por ser autónomos y proactivos (Holtz, 2016).

Licona Meneses y Veytia Bucheli (2019) explican que el uso de TIC promueve cambios que van desde los diseños curriculares hasta la individualidad de los procesos de enseñanza y aprendizaje, generando nuevos escenarios de aprendizaje innovadores como el m-learning o b-learning, las actividades ubicuas, aulas virtuales de aprendizaje, entre otros. Estos ambientes no solo son valorados por los estudiantes, sino que se plantean como alternativas a los clásicos materiales impresos de lectura, que perciben como desactualizados, frente a la vertiginosa producción de conocimiento, disponible y accesible desde la web en tiempo real:

"Los materiales son edición antigua, desactualizados (...)" (Estudiante 27, C.M.). "El material de estudio suele dificultar un poco, ya que la mayoría son viejos, a veces mal sacados, o están subrayados; lo que dificulta un poco en la forma de ser abordados" (Estudiante 37, C.).

"En general son acordes al programa, pero considero que falta más acercamiento a las prácticas, al contacto con la realidad" (Estudiante 63, J.).

"No me favorecen los textos antiguos, que no presentan ejemplos y que se encuentran deteriorados y/o rayados por el paso del tiempo y por el uso de tantos alumnos" (Estudiante 74, L.).

Los estudiantes ponen sobre la mesa la necesidad de un cambio en las rutinas de aprendizaje, de contenidos, de procesos cognitivos para aprender y prácticas de enseñanza. Área y González (2015) sostienen que los libros de texto son los recursos didácticos hegemónicos de la educación moderna. En este sentido, explica que el libro de texto es un dispositivo caracterizado por nacer como consecuencia del movimiento enciclopedista bajo una lógica que responde a la cultura impresa. En él se plasmaron características específicas de la enciclopedia, como la organización y síntesis del conocimiento general y racional existente de diversas áreas o disciplinas, que posteriormente se trasladaron a la enseñanza.

En este punto, Aguilar (2012), menciona la necesaria transformación de las metodologías tradicionales en estrategias innovadoras y creativas que permitan la construcción de los aprendizajes de sus estudiantes, revolucionando el modo de obtener, manejar e interpretar información. Así también lo expresan los estudiantes:

"Apuntes claros, contextualizados; que sean en diferentes formatos, audiovisuales, visuales, digitalizados, formato papel, etc.” (S38).

"Lo que favorece que el material este digitalizado porque permite ir viendo que material se puede tener acorde al bolsillo de cada uno y el programa ir siguiendo acorde al material" (S42).

"Textos originales y fichas de estudio, pero manteniendo la relación entre ellas, que se presente realmente aquello que apunta a lo abordado en el momento. La ayuda de los profesores es necesaria para que motiven a leer el material" (S46).

"Particularmente en nuestra carrera hablando de un plan de estudios de 1998, que a mi parecer debería ser actualizado urgentemente porque tienen cosas que no son muy actuales y que no resultan significativas, son contenidos aislados y deberían modificarse" (S12).

Área y González (2015), hablan de una necesaria "metamorfosis digital de los materiales educativos" como aquella transformación que atraviesa el material didáctico en pos de una nueva generación de recursos o entornos destinados a su utilización pedagógica, que va más allá del cambio de soporte tecnológico. Implica, no sólo el paso del libro impreso al uso de herramientas y recursos digitales, sino una transformación mayor en el paradigma pedagógico. Mutación que supone nuevas 
prácticas organizativas y didácticas hacia el interior del aula, tanto procesos de enseñanza innovadores dirigidos al aprendizaje activo como una reconstrucción de la cultura educativa que responda a las necesidades de la sociedad digital. En relación a los cambios que refieren a la digitalización del material educativo, De Pablos, Colás, López Gracia y García-Lázaro (2019), mencionan que la transformación de su naturaleza implica cambios a nivel de producción, como distribución y consumo a través de plataformas y portales online, así como, en la producción y distribución de materiales entre profesores, y desarrollo de recursos educativos abiertos.

\section{Motivos para desvincularse y revincularse}

Dentro de esta categoría pretendemos incluir aquellos vínculos, acciones y estrategias vinculadas al estudiante, que posibilitan u obstaculizan su vinculación con la carrera universitaria elegida, así como las estrategias que utilizan para revincularse con sus estudios. Durante el período en el que los estudiantes permanecen en el ámbito universitario, pueden experimentar una o varias fases de un fenómeno compuesto por las siguientes situaciones: recursar todo un año de la carrera o en el cursado de algunas materias, prolongación del tiempo requerido para obtener la titulación, cambio de carrera o bajas calificaciones académicas, abandono y posterior reingreso o deserción sin continuar los estudios superiores (González, 2005; Cabrera, Tomás, Álvarez y González, 2006; Desjardins, Ahlburgb \& McCall, 2006). Estos factores se ligan a la actualidad con un proceso que se conoce como desvinculación educativa, el cual se produce por la acumulación de elementos que originan cierto grado de alejamiento respecto a la escala de valores, pautas de actuación o los símbolos de identificación con la institución educativa (Fernández Enguita, Mena y Riviere, 2010).

Glasman (2000) en su estudio establece una distinción que sirve para iniciar el análisis.

Menciona dos grupos de estudiantes, los que se descuelgan definitivamente, refiriéndose a aquellos estudiantes que abandonan los estudios tempranamente, pero a su vez califica a otro sector de estudiantes como los que se descuelgan sin moverse de su sitio, es decir, se desvinculan sin abandonar los estudios, cuyos motivos están ligados a no romper con lo que, dentro del entorno académico o familiar, sigue siendo lo esperado según las expectativas construidas. Sobre este segundo grupo, compartimos los motivos que expresan los estudiantes que conforman la muestra en estudio.

La desvinculación, sin abandonar temporal o definitivamente la universidad, se puede observar en las siguientes expresiones de los estudiantes:

"La ayuda tiene que ver con la constancia para poder avanzar, y en ese avance es que uno se va descubriendo como estudiantes, va conociendo fortalezas y debilidades, así más simples de comprender y recordar. Por otro lado, no todo plan resulta como esperábamos ni todo esfuerzo obtiene el resultado buscado, las exigencias académicas, las formalidades, las normas universitarias, pueden afectar de manera negativa el cómo nos vemos como estudiantes y como nos visualizamos a futuros. Ante estas 'crisis' es que se definirá como continuaremos, si lo haremos y si salimos enriquecidos de ese obstáculo, seguramente esto afianza más nuestro compromiso en continuar y culminar la carrera" (S5).

"Sí, la exigencia es mucha y a veces no damos abasto en cuanto energía y cansancio con todo lo que tenemos que hacer para cumplir con las asignaturas. Esto me ha llevado muchas veces a replantearme si realmente seguir o dejar todo. Siempre conté con el apoyo de familiares y amigos que han ayudado a no bajar los brazos y seguir con lo que realmente quiero hacer (S2)".

“... particularmente, que soy de otro lugar me ha afectado el hecho de no tener a mi familia o amigos cerca y tener que adaptarme a algo nuevo, esas situaciones de que solo ves a tu familia los fines de semana, me ha llevado por ahí a querer dejar, o a 
desengancharme, pero pienso que todo esfuerzo tiene su fruto y por más que me falten varios años, pienso que puedo continuar y terminar lo que yo elegí hacer" (S24).

"Considero que las estrategias que he ido utilizando no han sido del todo adecuadas, por lo tanto, me cuesta también llevar la carrera al día, muchas veces el desaprobar parciales, finales o no lograr el objetivo, me llevan a sentirme fracasada y querer dejar todo. Pero también pienso en todo el esfuerzo no sólo el que hace mi familia para que yo pueda estudiar acá, como mi esfuerzo por tener que estar lejos de todo lo que quiero y eso me lleva a continuar con mis estudios" (S22).

Jiménez Herrera (2016), reconoce causas culturales y demográficas asociadas a los procesos de desvinculación, como el distanciamiento familiar, la adaptación a un nuevo lugar-hogar y el establecimiento de nuevos vínculos, con pares, docentes y con la misma. A lo que Castaño, Gallón, Gómez y Vázquez (2006), suman variables de tipo académicas, al expresar que a medida que aumentan los exámenes aprobados, es menor la tendencia a retirarse del sistema educativo, mientras que cuanto más se desaprueba la tendencia se inclina hacia el abandono.

La decisión del estudiante de comprometerse con sus estudios está no sólo influida por su grado de interacción académica- social, sino también por las actividades educativas que se diseñan para incrementar la posibilidad de que los estudiantes aprendan y logren avances significativos (Astin, 1993; Pascarella \& Terenzini, 2005). La ausencia de experiencias educativas que inspiren la participación y el interés promueven la presencia de estudiantes que están mentalmente ausentes en la clase. Así lo expresan:

"Si la clase me resulta aburrida, me quedo en silencio y trato de comprender o realizar avances de otras materias, o retirarme antes de que termine" (S2).

"Sí, me es muy fácil distraer en clase, no el hecho de conversar con otros compañeros, pero si de por ahí estar pensando en otras cosas" (S3).

"No molesto a mis compañeros, pero si suelo distraerme y ponerme a charlar con mis compañeros más allegados de otra temática que nada tiene que ver con lo que se está exponiendo" (S4).

Frente a uno u otro motivo, los estudiantes buscan estrategias para volver a reencauzar las energías y el compromiso con los estudios. Localizan las ayudas principalmente en otros, ya sean pares o docentes:

"Considero que es posible remontar todo el tiempo ante momentos de recaída, consiguiendo las energías necesarias en cada situación para continuar. Generando nuevos vínculos, antes que nada, logrando organizarse ante el material y los conceptos que nos brinda la carrera, y manteniendo todo el tiempo la comunicación con los docentes que forman el andamiaje necesario a la hora de adquirir nuevos aprendizajes" (E18).

"Sí, en realidad las acciones de compañerismo, el sentirnos cómodos en la universidad y contar con el apoyo de estudiantes y docentes, creo que influyen mucho en nuestro avance. Por otro lado, la falta de energía para seguir en algunos momentos suele estar presente, quizás en situaciones en donde desaprobamos o sentimos que no podemos aprender (...) porque no logramos avanzar, o que no contamos con el apoyo que necesitamos y al sentirse solos esto desanima y creo que de esa forma si se termina abandonando la carrera" (E25).

Lam, Wong, Yang y Liu (2012), mencionan que las relaciones interpersonales y los soportes por parte de los docentes, pares y familia son destacados entre los estudiantes que tienden a comprometerse con sus estudios. Onrubia, Colomina y Engel (2008) sostienen que no consiste solo en dejar que los alumnos interactúen o promover la interacción entre ellos para lograr un impacto favorable, sino que hay que pensar y repensar en cómo diseñar y planificar la interacción que se 
pretende llevar a cabo para que éstas puedan tener un impacto tanto cognitivo como social en los estudiantes. Se considera que la implicación de los padres es beneficiosa para los hijos cuando apoya el desarrollo de la autonomía, se centra en el proceso y comprenda afectos positivos (Rego, Otero y Moledo, 2016).

Asimismo, se visualiza entre los alumnos del estudio comportamientos proactivos que lo ayudan a seguir comprometidos o revincularse con sus estudios superiores, así lo expresan:

"Creo que a lo largo de la carrera hay muchas cuestiones que influyen en la motivación y permanencia y hay altibajos. Pero una de las cosas fundamentales es la convicción, si uno sabe lo que quiere y por qué lo quiere, debe aferrarse a eso y buscar las formas, las estrategias, el apoyo necesario y también saber enfrentar y pasar por las malas situaciones y seguir para lograr la meta" (E13).

"Muchas veces las estrategias que empleamos no son las adecuadas al objetivo que se persigue y eso nos lleva a un sentimiento de fracaso, de no seguir" (E15).

La dimensión agéntica, está ligada a la habilidad del alumno para establecer metas propias y constructivas en el proceso activo de aprendizaje. Este componente implica ser responsable de las propias decisiones y actuar de manera autónoma. Proactivamente modifican el contexto o la situación que los desvincula y los mueve del interés y la participación por sus estudios, para volver a comprometerse. Ese retomar, está cargado por los motivos, los objetivos y los propósitos que los estudiantes se formulan para desplegar "esa energía en acción”, que implica estar involucrado en los estudios para alcanzar esas metas (Reeve, Cheon \& Jang, 2019), incluso obtener el título de grado.

\section{Consideraciones finales}

En este estudio se propuso describir los motivos que los estudiantes universitarios construyen para comprometerse, así como las acciones y comportamientos que desarrollan cuando se desvinculan y vuelven a comprometerse. Partimos de considerar la necesidad de comprender el compromiso como un metaconstructo modificable y situado que, como continuo, fluctúa a lo largo de los procesos de aprendizaje de los estudiantes universitarios, en función de las percepciones que van teniendo de sus estudios, las clases, los docentes, sus esfuerzos y los apoyos recibidos o buscados. Factores personales y contextuales que, a modo de energía, impulsan las acciones y comportamientos de los estudiantes hacia uno u otro lado del péndulo, para definir los procesos de vinculación y desvinculación académica.

$\mathrm{El}$ análisis de las respuestas de los alumnos nos permite apreciar ciertas inquietudes vinculadas a por qué el estudiante manifiesta poca o escasa participación durante las clases y el cursado de carreras universitarias, cuáles son los motivos que los alumnos aluden a sus procesos de desvinculación en las aulas o frente a sus estudios, y qué objetivos o acciones personales llevan a cabo para volver a comprometerse.

Encontramos que los motivos que comprometen a los estudiantes se inclinan hacia la necesaria trasformación y extensión de los límites físicos del aula, definiendo prácticas educativas en contextos que tiendan a incluir actividades de extensión y vinculación social, junto a la incorporación de nuevos recursos digitales y tecnológicos para enseñar. También, hallamos que los motivos que desvinculan son múltiples, asociados al plan de estudio, al cursado de la carrera, y a la configuración de la clase. En una u otra circunstancias contar con apoyos, de docentes, familia y amigos es crucial, pero sobre todo generar nuevos vínculos en torno al contenido, las estrategias y los otros para alcanzar la meta -terminar de cursar, recibirse, rendir un parcial-, como recursos que facilita el volver a comprometerse con los estudios. 
Otras investigaciones muestran que los resultados del presente estudio coinciden en varios puntos. Al respecto, Harnish, Bridges, Denillo y Flaherty (2014), encuentran que las actividades que los estudiantes pueden aplicar y relacionar con problemas sociales tales como prácticas, pasantías y experiencias de campo, están ligadas a sus intereses y promueven el compromiso. Asimismo, reconocen dos características críticas en la implicación de algunos. La primera relacionada al tiempo y el esfuerzo que dedican a sus estudios y actividades educativas, y la segunda referida a cómo las instituciones despliegan recursos y servicios de apoyo, en términos de organizaciones de planes de estudio, para promover el compromiso de los estudiantes. En tal sentido, las conclusiones del estudio desarrollado por Harnish y Snider (2013), coinciden en que los motivos para involucrarse en actividades de participación comunitaria pueden ser personales o sociales.

Pike y Kuh (2005), exponen entre sus resultados que tanto el trabajo colaborativo como mediado por las tecnológicas de la información y la comunicación es coincidente con el perfil de estudiantes comprometidos o aquellos rasgos que las instituciones educativas contemplan para diseñar la propuesta educativa; asimismo, detallan que los estudiantes se ven involucrados cuando los docentes y los entornos educativos ofrecen experiencias de aprendizaje diversas, incluyendo interacciones por fuera de la clase y relaciones interpersonales de calidad. González González y Cutanda López (2015), en una revisión teórica expresan que la no implicación se produce como consecuencia de dificultades que van marcando las trayectorias educativas de los estudiantes, asociadas a niveles bajo de logro o reiterados aplazos, remarcando lo fundamental que es aumentar los apoyos sociales positivos de los alumnos con la institución para atenuar los niveles de desvinculación.

En síntesis, los resultados muestran que la complejidad del compromiso es inherente a las individualidades de los estudiantes. Si bien las percepciones sobre los estudios superiores son particulares a cada sujeto, hay una construcción conjunta que permite identificar motivos compartidos. Al respecto, Zepke, (2011), entiende que el compromiso, desde la teoría de la complejidad, es una red dinámica, no jerárquica, en la cual los factores son distintos, aunque están conectados. En la misma línea, Sauve y Schonert-Reichl (2019), reconocen que el desarrollo humano es un proceso relacional bidireccional, individuo-contexto, en el cual encontramos dimensiones relacionadas al sujeto, pero también múltiples factores dentro de la ecología social, que contribuyen al desarrollo.

Los resultados son un claro aporte para profundizar sobre un aspecto que muchas veces fue relegado al indagar sólo los motivos que desvinculan a los estudiantes que están a punto de desertar. $\mathrm{Al}$ respecto, McInnis (2001) puntualizó la falta de estudios encaminados a conocer los motivos, los valores y las expectativas cambiantes de los estudiantes universitarios en relación con sus niveles de compromiso, advirtiendo sobre el riesgo de sólo centrarse en aquellos que están al límite de fracasar, perdiendo las vivencias previas durante el cursado que pudieran evitar mucho antes la salida del sistema educativo.

En tal sentido, el estudio desarrollado aporta datos concretos para tener una comprensión más clara del compromiso de los estudiantes y sus interacciones con el contexto educativo, lo que nos habilita a mejorar, a futuro, las experiencias, los planes de estudio y los resultados educativos. Ciertamente, se necesitan nuevas investigaciones para explorar más a fondo los motivos para vincularse-desvincularse-revincularse en educación superior, para contar con información que, situada a la realidad de los jóvenes, nos permitan habilitar acciones y programas tendentes a fortalecer el interés, la participación y la permanencia en las carreras de grado.

Concretamente, el compromiso agente suponemos tiene un rol clave en el diseño de contextos educativos, contemplando los aportes de la teoría de la autodeterminación, aumentando la autonomía de los estudiantes (Jang, Kim \& Reeve, 2016), explicitando intereses, expectativas, valores y metas que guíen sus estudios y amplifiquen sus intereses y niveles de participación en la creación 
de un contexto de aprendizaje para involucrase, es un dato a seguir profundizando en próximos estudios.

\section{Referencias}

Aguilar, M. (2012). Aprendizaje y Tecnologías de Información y Comunicación: Hacia nuevos escenarios educativos. Revista Latinoamericana de Ciencias Sociales, Niñezy Juventud, 10(2), 801-811.

Appleton, J. J., Cristenson, S. L., Kin, D., \& Reschly, A. L. (2006). Measuring cognitive and psychological engagement: validation of the student engagement instrument. Journal of School Psychology, 44, 427-445.

Área, M. y González, C. S. (2015). De la enseñanza con libros de texto al aprendizaje en espacios online gamificados. Educatio Siglo XXI, 33(3),15-38. https://doi.org/10.6018/j/240791

Astin (1993). What matters in College? Four critical years revisited. San Francisco, CA: Jossey-Bass.

Bandura, A. (2006). Toward a psychology of human agency. Perspectives on Psychological Science, $1(2), 164$ 180.

Brigman, G., Wells, C., Webb, L., Villares, E., Carey, J. C. \& Harrington, K. (2015). Psychometric properties and confirmatory factor analysis of the Student Engagement in School Success Skills. Measurement and Evaluation in Counseling and Development, 48(1), 3-14.

Cabrera, L., Tomás, J., Álvarez, P. y González, M. (2006). El problema del abandono de los estudios universitarios. RELIEVE, 12(2), 171-203.

Castaño, E., Gallón, S., Gómez, K. y Vázquez, J. (2006). Análisis de los factores asociados a la deserción y graduación estudiantil universitaria. Lecturas de Economía, 65, 9-36.

Casuso-Holgado, M. J., Cuesta-Vargas, A. I., Moreno-Morales, N., Labajos-Manzanares, M. T., BarónLópez, F. J. \& Vega-Cuesta, M. (2013). The association between academic engagement and achievement in health sciences students. BMC Medical Education, 13, 33. https://doi.org/10.1186/1472-6920-13-33

Cecchi, N. H., Pérez, D. A. y Sanllorenti, O. (2013). El compromiso social de la Universidad Latinoamericana del Siglo XXI: entre el debate y la acción. Buenos Aires: IEC - CONADU.

Colás Bravo, P., González Ramírez, T. y Reyes De Cózar, S. (2015). Características de las aulas universitarias que generan engagement desde la perspectiva de los estudiantes. En AIDIPE (Ed.), Investigar con y para la sociedad (Vol. 2, pp. 691-703). Cádiz, España: Bubok

Connell, J. P. (1990). Context, self and action: A motivational analysis of self-system processes across the life span. En D. Cicchetti y M. Beeghly (Eds.), The self in transition: Infancy to childhood (pp. 61-97). Chicago: University of Chicago Press.

Coulon, A. (1999). An instrument d' affiliation intellectuelle. L'enseignement de lamethodologie documentaire. París: BBF.

De Souza Santos, B. (2005). La universidad en el siglo XXI. Para una reforma democrática y emancipadora de la universidad. Buenos Aires: LPP- Miño y Dávila Editores.

DesJardins, S., Ahlburg, D. \& McCall, B. (2006). An Integrated Model of Application, Admission, Enrollment, and Financial Aid. Journal of Higher Education, 77(3), 381-429.

De Pablos, J.M., Colás, M.P., López Gracia, A. y García-Lázaro, I. (2019). Uses of digital platforms in Higher Education from the perspectives of the educational research. REDU. Revista de Docencia Universitaria, 17(1), 59-72.

Fernández Enguita, M., Mena, L., y Riviere, G. (2010). El fracaso y abandono escolar en España. Barcelona: Fundación La Caixa.

Finn, J. D. (1989). Withdrawing from school. Review of Educational Research, 59(2), 117-142. 
Finn, J. D. (1993). School Engagement \& Students at Risk. Washington DC: National Center for Education Statistics.

Fredricks, J., Blumenfeld, P. \& Paris, A. (2004). School Engagement: Potential of the concept, State of the Evidence. Review of Educational Research, 74(1), 59-109.

Glasman, D. (2000). Le décrochage scolaire: une question sociale et institutionnelle. VilleÉcoleIntegration Enjeux, 122, 10-25.

González González, M. T. y Cutanda Lopéz, T. (2015). La formación del profesorado y la implicación (engagement) de los alumnos en su aprendizaje. Revista Iberoamericana de Educación, 69(2), 9-24.

González, M. T. (2005). El absentismo y el abandono: Una forma de exclusión escolar. Profesorado. Revista de curriculum y formación del profesorado, 9(1), 1-13.

Graumann, C. F. (1971). Fundamentos de Psicología. Motivación. Madrid: Morata.

Harnish, R., Bridges, R., Denillo A. \& Flaherty, M. (2014). Engaging Students in Applied Social Psychology. In R. L. Miller, E. Balcetis, S. R. Burns, D. B. Daniel, B. K. Saville \& W. D. Woody (Eds.), Promoting student engagement (Vol. 2, pp. 38-43). Society for the Teaching of Psychology.

Harnish, R. J. y Snider, K. J. (2013). Understanding Community Engagement Motives: A "Functional" Approach". Journal of Regional Engagement, 2(2), 132-152.

Hofkens, T. y Ruzek, E. (2019). Measuring Student Engagement to Inform Effective Interventions in Schools. In J. Fredricks, A. Reschly \& S. Christenson (Edits,), Handbook of Student Engagement Interventions (pp. 309-324). London: Academic Press.

Holtz, D. (2016). 'Millennials', 'generación Z' y educación superior. Disponible en https://www.milenio.com/opinion/dieter-holtz/columna-dieter-holtz/millennialsgeneracion-z-y-educacion-superior. Consultado en 28/02/2020 a las 10:59.

Jang, H., Kim, E. \& Reeve, J. (2016). Why students become more engaged or more disengaged during the semester: A self-determination theory dual-process model. Learning and Instruction, 43, 2738. http://dx.doi.org/10.1016/j.learninstruc.2016.01.002

Jiménez Herrera, L. G. (2016). Reflexiones teóricas del fenómeno de repitencia, prolongación, abandono y deserción de estudiantes universitarios. Pensamiento Actual, 16(227), 261-269.

Lam, S., Wong, B., Yang, H. \& Liu, Y. (2012). Understanding student engagement with a contextual model. En S. Christenson, A. Reschly \& C. Wylie (Eds.), Handbook Research on Student Engagement (pp. 403-419). New York. Springer.

Licona Meneses, K. y Veytia Bucheli, M. G. (2019). El empleo de las TIC en la educación superior. Educando para educar, 37, 91-99.

Macchiarola, V., Martini, C. y Montebelli, A. E. (2012). Prácticas socio comunitarias en el trayecto formativo del profesional de Educación Especial. Revista RUEDES. Red Universitaria de Educación Especial, 2(3), 61-77.

Martin, A. J. (2008). Enhancing student motivation and engagement: The effects of a multidimensional intervention. Contemporary Educational Psychology, 33, 239-269.

Martínez, I. y Salanova, M. (2003). Niveles de burnout y engagement en estudiantes universitarios. Relación con el desempeño y desarrollo profesional. Revista de Educación, 330, 361-384

Martínez, J. S. (2007). Fracaso escolar, clase social y política educativa. El Viejo Topo, 238, 45-49.

Martínez, J. S. (2009). Fracaso escolar, PISA y la difícil ESO. Revista de la Asociación de Sociología de la Educación (RASE), 2(1), 56-85.

McInnis, C. (2001). Signs of disengagement? The changing undergraduate experience in Australian universities: inaugural professorial lecture. Melbourne: Centre for the Study of Higher Education.

Montes Ruiz, A. (2016). En la encrucijada. Factores de desenganche y reenganche educativo en la trayectoria de seis jóvenes en Cataluña (España). Inguruak, 61,1-22. 
Newmann, F. M., Wehlage, G. G., \& Lamborn, S. D. (1992). The significance and sources of student engagement. En D. M. Newmann (Ed.), Student engagement and achievement in american secondary schools (pp. 11-39). New York: Teachers College Press.

Onrubia, J., Colomina, R. y Engel, A. (2008). Los entornos virtuales de aprendizaje basados en el trabajo en grupo y el aprendizaje colaborativo. En C. Coll (Ed.), Psicología de la educación virtual: aprender y enseñar con las tecnologias de la información y de la comunicación (pp. 132-152). Madrid: Morata.

Pascarella, E. \& Terenzini, P. (2005). How college affects students: Findings and insights from twenty years of research. San Francisco: Jossey-Bass.

Pike, G. R. \& Kuh, G. D. (2005). A typology of student engagement for American colleges and universities. Research in Higher Education, 46(2), 185-209.

Reeve, J. (2012). A self-determination theory perspective on Student engagement. En S. L. Christenson, A. L. Reschly \& C. Wylie (Eds.), Handbook of research on Student engagement (pp. 149172). New York: Springer.

Reeve, J., Cheon, S. \& Jang, H. R. (2019). A Teacher-Focused Intervention to Enhance Students' Classroom Engagement. In J. Fredricks, A. Reschly \& S. Christenson (Eds.), Handbook of Student Engagement Interventions (pp. 87-100). London: Academic Press.

Rego, M., Otero, A. y Moledo, M. (2016). ¿Puede la implicación de los padres mejorar el estudio de sus hijos en la escuela? La evidencia de un programa pedagógico. Estudios sobre Educación, 30, 9-3.

Rigo, D. y Donolo, D. (2019). Análisis de un modelo integrador del compromiso escolar: relaciones entre variables situacionales, escolares, sociales y personales en alumnos de nivel primario de $\begin{array}{llllll}\text { educación. } & \text { Propósitos } & y & \text { Representaciones, } & 7 & \text { (SPE), }\end{array}$ http://dx.doi.org/10.20511/pyr2019.v7nSPE.316

Ríos, D., Bozzo, N., Marchant, J. y Fernández, P. (2010). Factores que incidenen el clima de aula universitario. Revista Latinoamericana de Estudios Educativos, XL(3-4), 105-126.

Rodríguez, P., Brum, L., Correa, A., Laporta, P., Cantieri, R., Núñez, C., Verrastro, N. y De León, G. (2014). La desvinculación en la primera generación de estudiantes de un programa innovador de la Universidad de la República, Uruguay. Revista de la educación superior, 43(170), 113-134.

Sauve, J. A. \& Schonert-Reichl, K. A. (2019). Creating Caring Classroom and School Communities: Lessons Learned from Social and Emotional Learning Programs and Practices. In J. Fredricks, A. Reschly \& S. Christenson (Eds.), Handbook of Student Engagement Interventions (pp. 279-295). London: Academic Press.

Skinner, E. A., Kinderman, T. A. \& Furrer, C. J. (2009). A motivational perspective on engagement and disaffection: Conceptualization and assessment of children's behavioral and emotional participation in academic activities in the classroom. Educational and Psychological Measurement, 69, 493-525.

Vasilachis, I. (2007). Estrategias de investigación cualitativa. Gedisa: Barcelona.

Voelkl, K. E. (1997). Identification with school. American Journal of Education, 105, 294- 318.

Wang, M. T. \& Peck, S. C. (2013). Adolescent educational success and mental health vary across school engagement profiles. Developmental Psychology, 49(7), 1266-1276.

Windham, C. (2005). The Student's Perspective. In D. Oblinger y J. Oblinger (Eds.), Educating the Net generation (pp. 5.1-5.16). Boulder, CO: EDUCAUSE. Disponible en: https://www.educause.edu/ir/library/pdf/pub7101e.pdf

Zepke, N. (2011). Non-institutional influences and student perceptions of success. Studies in Higher Education, 36, 227-42. 


\section{Información sobre las autoras}

Autora: Daiana Yamila Rigo

Institución: Instituto De Investigaciones Sociales, Territoriales y Educativas Consejo Nacional de Investigaciones Científicas y Técnicas. Universidad Nacional de Río Cuarto Email: daianarigo@hotmail.com

ORCID: https://orcid.org/0000-0003-0312-6429

Autora: Melina Irusta

Institución: Centro Educativo Terapéutico Kalen

Email: melina_90_4@hotmail.com

ORCID: https://orcid.org/0000-0002-6116-6704

Autora: Gisela Bechero

Institución: Centro Educativo Terapéutico Kalen

Email: andypety_gise@hotmail.com

ORCID: https://orcid.org/0000-0002-0590-4303

Autora: Stefania Amaya

Institución: Universidad Nacional de Río Cuarto

Email: stefaniasamaya@gmail.com

ORCID: https://orcid.org/0000-0003-0845-2151 


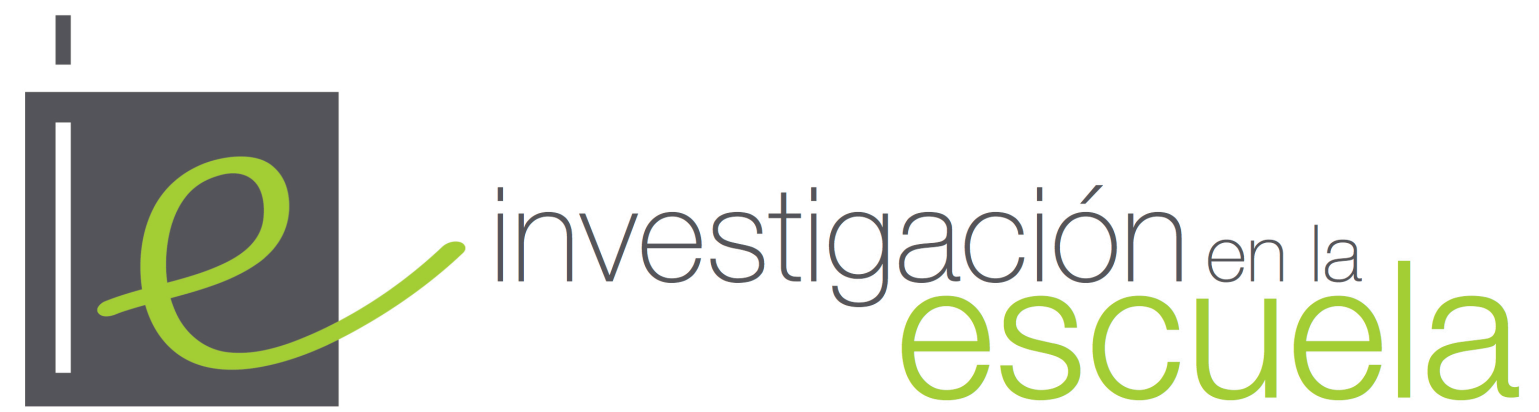

Revista académica evaluada por pares y de acceso abierto

Número 100

30 de abril de 2020

ISSN 2443-9991

\section{c) () (2)}

Esta obra está bajo una licencia Creative Commons. Los/as lectores/as pueden compartir, copiar y redistribuir el material en cualquier medio o formato, así como adaptar, remezclar, transformar y construir a partir del material para cualquier propósito, incluso comercialmente. Para ello, deben de hacerlo bajo los siguientes términos: dando crédito de forma adecuada, brindando un enlace a la licencia e indicando si se han realizado cambios. Si se remezcla, transforma o crea a partir del material, debe distribuir su contribución bajo la misma licencia del original.

Más detalles de la licencia de CreativeCommons se encuentran en https://creativecommons.org/licenses/by-sa/4.0/deed.es

Cualquier otro uso debe ser aprobado en conjunto por el autor/es, o Investigación en la Escuela.

Uิ?

Revista Editada por la Universidad de Sevilla. https://editorial.us.es/es/revistainvestigacion-en-la-escuela

Por errores y sugerencias contacte a investigacionenlaescuela@us.es

La revista Investigación en la Escuela desde su origen en 1987 hasta su no 87 (2015) fue editada por Díada Editora. 
